\title{
Die ethische Relevanz der ästhetischen Kontemplation
}

\author{
von Margit Ruffing \\ Johannes Gutenberg-Universität - Mainz (Deutschland)
}

\begin{abstract}
ZUSAMMENFASSUNG
Schopenhauers hat seine Philosophie von der Welt als Wille und Vorstellung als Gesamtsystem in vier Teilen durchgeführt: Die Einheit des Wesens der Welt wird sichtbar im Ausgang vom willens- und zweckbestimmten natürlichen Erkennen (1. Buch) über die Erkenntnis der Erscheinungshaftigkeit (Objektität) des Willens am eigenen Leib (2. Buch) und die Möglichkeit der willensfreien, idealen Erkenntnis in der ästhetischen Kontemplation (3. Buch) bis zur Durchschauung des principium individuationis, d.h. der Selbsterkenntnis des Willens (4. Buch). In diesem Beitrag wird die Theorie der ästhetischen Kontemplation als eine Philosophie des Bewusstseins aufgefasst, die die zentrale Bedeutung des dritten Buches für das Gesamtsystem legitimiert. Dabei steht die spezifische Geisteshaltung der Besonnenheit im Zentrum der Betrachtungen, insofern sie Erkenntnisbedingung und Kennzeichen der menschlichen Moralität ist. In der Realität der ästhetischen Kontemplation zeigt sich demnach schon die Moralitätsfähigkeit des menschlichen Bewusstseins als Bedingung der Möglichkeit moralischen Handelns.
\end{abstract}

\begin{abstract}
Schopenhauer organized his philosophy of the world as will and representationsystematically, dividing the work into four sections. The unity of the essence of the worldarises in different ways, according to the four books of the main work, by means of ournatural volitional and purposive cognition (book 1), the recognition of the "objectity" of thewill, experienced on the own body (book 2), the possibility of ideal cognition, freed fromthe will, in aesthetic contemplation (book 3) up to the understanding ("Durchschauung")of the principium individuationis: the self-knowledge of the will (book 4). In this article,the theory of aesthetic contemplation is regarded as a philosophy of consciousness, whichlegitimizes the central importance of the third Book for Schopenhauer's philosophical systemas a whole. Our central reflections refer to the specific mental attitude of "Besonnenheit"insofar as it is both a condition of knowledge and a characteristic of human morality. Hencethe human capacity for morality is present in actual aesthetic contemplation - as a conditionof the possibility of acting morally.
\end{abstract}

\section{EinleitendeBemerkungenzum System der Welt alsWille und Vorstellung}

SchopenhauersPhilosophie, die dieeine Welt alsWille und Vorstellungbetrachtet und erklärt, will den einenGedanken - dass die Welt Selbsterkenntnis des Willenssei - begreifbarmachen. ${ }^{1}$ Siebildetdaherein System, und sie muss estun, denn

die wahrePhilosophieentsprichtnachSchopenhauerseigenerAnsichteinemorganischenGa ${ }^{1}$ Siehedazuauch: Rudolf Malter: Der eineGedanke. HinführungzurPhilosophie
Schopenhauers.Darmstadt: WissenschaftlicheBuchgesellschaft. 2. Aufl. $2010\left[{ }^{1} 1988\right]$. 
nzen, nämlichdemLebenselbst und seiner Bedeutung, die von ihm, demPhilosophen, erfasst, in Begriffenausgedrückt und in eine - systematische Form gebrachtwurde. Und diesedientdazu, die philosophischeEinsicht in das Wesen der Welt mitzuteilen, sie der Erkenntnisandererzugänglichzumachen. ${ }^{2}$ Das System ist also für Schopenhauer weniger die Sacheselbstals die von ihmals optimal erachteteDarstellungsform des einenGedankens der Welt alsSelbsterkenntnis, als „Autopräsentation“ desWillens. Der optimalenDarstellungsformentspricht die dualistischeStruktur des Schopenhauerischen Systems, ${ }^{3}$ die Aufteilung in je zweiBücher, die von der Vorstellung, der Erkenntnis, und demVorgestellten, demErkannten, handeln, einmalunterdemSatzvomGrundestehend, einmalalsunabhängig von demselbenanzusehen. Genau betrachtet haben wir es bei Schopenhauers „Welt als Wille und Vorstellung“ daher immer mit einer Theorie des Erkennens zu tun: Im ersten Buch geht es um die „alltägliche“ und die wissenschaftliche Erkenntnis, die alles in Beziehungen von Ursache und Wirkung ordnet; das zweite Buch erläutert eine toto genere andersartige Erkenntnis, nämlich die gefühlte Gewissheit, dass das eigene Wesen nicht aufgeht in der Kausalität der Natur, d.h. die Einsicht in das wesentlich Willenshafte unserer Natur, und es erläutert, dass diese sich auf die gesamte Welt (per Analogieschluss) übertragen lässt; das dritte Buch spricht erneut von einer ganz anderen „Erkenntnißart“, der ästhetischen Kontemplation, und das vierte Buch behandelt schließlich die vollendete Selbsterkenntnis des Willens, die praktische Konsequenzen hat, indem sie dem Menschen eine gegen den naturhaft egoistischen Willen gerichtete moralische Einstellung und nicht-egoistisches, sogar altruistisches moralisches Handeln ermöglicht.

Mit anderen Worten ausgedrückt: Den vier Büchern, in denen Schopenhauer seine Philosophie systematisch entwickelt, liegen lediglich zwei Erkenntnisfragen zu Grunde - die Frage nach dem Was der Erkenntnis, dem Wesen, der Bedeutung der Welt, und die Frage nach dem Zugang zum Wissen des Wesentlichen, nach dem Wie der Erkenntnis. Antwort auf die erste Frage gibt bekanntermaßen das zweite Buch mit der Metaphysik des Willens, deren praktischer Aspekt im vierten Buch

\footnotetext{
${ }^{2}$ Dazuäußertsich Schopenhauer anverschiedenenStellen seines Werkes, s. besonders die Vorredezur 2. Auflage der Welt alsWille und Vorstellung, und W I, § 15, dort 98-99.

${ }^{3}$ Das Verhältnis von dualistischer Form und inhaltlicherEinheithabeichanandererStellebehandelt, s. „Die Duplizitätsstruktur des Bewußtseinsbei Schopenhauer und C. G. Jung, oder: 1+1=1“. In: 86. Schopenhauer-Jahrbuch (Würzburg) 2005, 195-212.
} 
als Mitleidsethik dargestellt wird. Das, was die Beantwortung der Frage nach dem Wesen aber erst ermöglicht, das systematisch entscheidende und alle vier Bücher verbindende Moment ist allerdings die Frage nach dem Wie, oder kantisch gesprochen, die nach den Bedingungen der Möglichkeit von Erkenntnis. Auch wenn sich Schopenhauers erkenntnistheoretische Ausführungen wie allgemein bekannt hauptsächlich im ersten befinden, lässt sich diese Frage m. E. erst vom dritten Buch her wirklich beantworten, auch im Hinblick auf dessen „Übergangsstellung“ zwischen erster, gefühlter, unvollkommener Willenserkenntnis und dem vollständigen Durchschauen des principium individuationis, zwischen Metaphysik und Ethik, provokativ gesagt: zwischen theoretischer und praktischer Vernunft. Schopenhauers Theorie der ästhetischen Kontemplation ist mehr als eine Philosophie der Künste: sie ist auch und zugleich eine Philosophie des Bewusstseins, wobei letztere die zentrale Bedeutung des dritten Buches für das Gesamtsystem legitimiert. ${ }^{4}$

Das dritte Buch der Welt als Wille und Vorstellung wurde allerdings, wie die Rezeptionsgeschichte zeigt, oft aus seinem systematischen Zusammenhang herausgelöst und publikumswirksam als Kunsttheorie, Ideenlehre, Lehre vom Genie interpretiert und rezipiert. ${ }^{5}$ Doch nimmt es meiner Auffassung nach nicht dieser Aspekte wegen eine besondere Stellung ein, sondern deshalb, weil es von einer besonderen Geisteshaltung handelt, die zur Kunst als Erkenntnisart ebenso gehört wie zum moralischen Bewusstsein: ich meine damit das, was Schopenhauer „Besonnenheit“ nennt: Besonnenheit meint Erkenntnisbedingung und Kennzeichen der menschlichen Moralität. ${ }^{6}$

Die Frage nach der Beschaffenheit der Wesenserkenntnis und unserer Erkenntnis vermögen, die sie hervorbringt, steht demnach in engstem Zusammenhang mit der Frage der Moralitätsfähigkeit des Menschen. Das möchte im folgenden Vortrag zu zeigen versuchen. Ich werde daher erst die ästhetische Kontemplation kurz vorstellen, wie sie von Schopenhauer im 3. Buch der WWV dargelegt wird, und

\footnotetext{
${ }^{4}$ DieseThematikstehtimZentrummeiner Dissertation: „,WillezurErkenntnis“. Die Selbsterkenntnis des Willens und die Idee des Menschen in der ästhetischenTheorie Arthur Schopenhauers, veröffentlichtim Online-Archiv der UniversitätsbibliothekMainzhttp://archimed.uni-mainz.de/pub/2002/0060/diss.pdf. ${ }^{5}$ Vgl. die BibliographiezurÄsthetikSchopenhauers, in: ,,WillezurErkenntnis", s. Anm. 4, 179-200.

${ }^{6}$ Eine umfassende Analyse des Bedeutungsspektrums von Besonnenheit bei Schopenhauer findet sich in einem Aufsatz von Matthias Koßler: „Zur Rolle der Besonnenheit in der Ästhetik Arthur Schopenhauers“. In: 83. Schopenhauer-Jahrbuch (Würzburg) 2002, 119-133.
} 
dann die ihr entsprechende Besonnenheit als intellektuelles Merkmal der menschlichen Moralität behandeln, um die ethische Relevanz der ästhetischen Kontemplation näher zu bestimmen.

\section{Die ästhetische Kontemplation}

Die Ideenlehre als solche ist bereits Bestandteil des 2. Buches; sie beschreibt die Überzeugung Schopenhauers, dass der Wille als metaphysisches Prinzip in die Vorstellung tritt und sich dieses „Objektivieren“ einer bestimmten Rangordnung oder Stufenfolge entsprechend vollzieht, wobei jede Stufe dabei die Urform, den Prototypen, d.h. die Idee der dazugehörigen Einzelobjektivationen bezeichnet. Die Ideen weisen auf ein Transzendieren der Erkenntnis nach dem Satz vom Grunde, auf eine neue Erkenntnisart hin. Um den besonderen Charakter der Idee hervorzuheben, vergleicht Schopenhauer sie mit dem Begriff: [ich zitiere]

„[...] der Begriff gleicht einem todten Behältniß, in welchem, was man hineingelegt hat, wirklich neben einander liegt, aus welchem sich aber auch nicht mehr herausnehmen läßt (durch analytische Urteile), als man hineingelegt hat (durch synthetische Reflexion): die Idee hingegen entwickelt in Dem, welcher sie gefaßt hat, Vorstellungen, die in Hinsicht auf den ihr gleichnamigen Begriff neu sind: sie gleicht einem lebendigen, sich entwickelnden, mit Zeugungskraft begabten Organismus, welcher hervorbringt, was nicht in ihm eingeschachtelt lag.“ (W I, 277)

Hier wird der Begriff, bildlich als „todtes Behältniß“ bezeichnet, der Idee gegenübergestellt, um das Lebendige, Dynamische, Kreative an ihr besonders hervorzuheben: Der Idee wird im Vergleich mit dem Ergebnis der abstrakten Vernunfttätigkeit, d.h. dem Begriff, ein innovatives Moment zugesprochen. Auf der einen Seite geht es darum, Wissen zu fixieren und kommunizierbar zu machen: am Ende dieser Bemühungen steht der Begriff. Auf der anderenSeitewird das, was der Begriffbezeichnet, erstals das, was esist, erkannt, wenn das erkennendeIndividuumdessenIdeeerfasst hat. Die Ideesteht in dieserErkenntnisweise am Anfang: sielöstBewusstseinsvorgängeimweitestenSinneaus, indemVorstellungenentstehen, „die in Hinsicht auf den ihr [der Idee, M. R.] gleichnamigenBegriffneusind“. SchopenhauersVergleich der Ideemiteinem „lebendigen, sichentwickelnden, mitZeugungskraftbegabtenOrganismus“ legtdeshalbmeinerAnsichtnachnahe, 
sienichtals Form oder „Behältniß“ der Erkenntnis, aberauchnichtalsbeliebigenInhalt, sondernalsKraftimSinne von Intensität des Erkenntnisinhaltsaufzufassen, die imBewusstseinetwasbewegt und verändert, um einWissen um die Sachehervorzubringen, das über den bloßenBegriffhinausgeht. Ichschlage also vor, diese von Schopenhauer alsnichtkausalcharakterisierteVorstellungals von räumlichen und zeitlichenBestimmungennichtbetroffene Kraft oderFähigkeit des Bewusstseinszudenken.

Der AusdruckWelt alsWille und VorstellunglässtsichmittelseinerKonzeption der als Kraft aufgefasstenIdee in Kürzefolgendermaßenauslegen: Die Welt istder Sachenach,Welt des WillensalsVorstellung“. Fürunsist die Welt, sofernwirsie, notwendigerweiseausgehendvomErscheinungshaften, ihremWes ennacherkennen, „Welt der VorstellungalsWille“ - den BlickaufsWesentliche am und innerhalb des Vorstellungshaftengewährtwiederum die Idee, siestehtdabeifürhöchsteWillensintensität in reinsterVorstellungshaftigkeit. So gesehenbezeugtsieeineTranszendenzleistung des Bewusstseins; allein die IdeeistGarantfür das Verstehen von philosophischenAussagenüber die „Welt alsWilleundVorstellung“. Die Erkenntnis des WillensalsWesen der Welt wirdsomit in der IdeezueinemlebendigenSelbst-Verständnis, in dem, Wollendes und ErkennendesalsungeschiedenesGanzesbewussterlebbar - und erlebt - werden.

Dieses Erleben, seiner Wirklichkeit und Möglichkeitnach, ist die ästhetischeKontemplation: alsGeschehen, alsBewusstseinsverfassung. Die ästhetischeKontemplationisteine Weise des Erkennens, das nicht auf den Begriffangewiesenzuseinscheint, bzw. nichtbeiihmstehenbleibt, sondern das Relationale $\quad$ Formaletranszendiert. In doppeltemSinnekannvomÜbergangscharakter der ästhetischenErkenntnisgesprochenwerden: 1.) Das praktischeErleben der Ideenschauist per se flüchtig und selbstimmerimÜbergang; 2.) die Position der Ideentheorieim 3. Buch der Welt alsWille und Vorstellungkann in theoretischerHinsichtalsVorbereitung auf die Ethikgelten, die jeneFlüchtigkeitzueinerdauerhaftenBewusstseinshaltunghinüberwindet.

Die ästhetischenKontemplationalsBewusstseinshaltungistdemErkennen und Verstehen des Wesentlichenangemessen, weilsie die Beschränkung auf die 
Einzelheit, das Individuelle, alsunwesentlich, nichtzumWesentlichen des Lebensgehörend, durchschaut; und zwar auf der Seite des Objekts, das betrachtetwird und in die Kontemplationführt, ebensowie auf der Seite des Subjekts, das in der Kontemplation die eigene - leidvolle - Beschränkung auf das Individuelleüberwindet. Die Selbstvergessenheit, von der Schopenhauer spricht, ${ }^{7}$ istnichtsanderesalseinVergessen des alltäglichenSelbstverständnisses, in dessenZentrumsichein "Ich“ befindet, von demausallesandereerkannt und beurteiltwird. Einsolches „Ich“ stehtimMittelpunktallerRelationen (nichtetwaimMittelpunkt der Welt!...), esist in dieseRelationeneingebunden und durchsiebedingt - was alleinschonalsUnfreiheit und Behinderung des eigenenWollensempfundenwird. VerstärktwirddieseEmpfindungdurch die Erkenntnis des Intellekts, dass die kausaleBedingtheit die gesamte Welt bestimmt und sozusagengefangenhält, aberauchdurch die Erfahrung, dass die konkurrierendenEinzelwillennichtzufriedenzustellensind und sichleidbringendgegeneinanderrichten.

Dem gegenüberbeschreibt Schopenhauer nun die ästhetischeKontemplationalsMöglichkeit, ausdieser Situation kurzfristigherauszutreten, sowohlhinsichtlich der intellektuellenErkenntnisalsauch die Leiderfahrungbetreffend. Schopenhauer beurteilt die ästhetischeErkenntnisweisealswesentliche, weilsie in der ästhetischenKontemplation das unmittelbareLeib- und Anschauungsbewusstseinzueineminteresselosen, zweckfreienBewusst-Sein von Schönheitoptimiert. Zudemwird die darinliegendeEinsicht in die Wesensgleichheit von Subjekt und Objekt in die nachträglichephilosophische Reflexion integriert: im 2. Buch, auf die Objektebezogen, ergibt das den Begriff der adäquatenObjektität, d.h. die Ideenlehre, im 4. Buchwird die Einsicht in die WesensgleichheitallerWillenserscheinungenzurGrundeinsicht des moralitätsfähigenVernunftwesens Mensch. Damit die ästhetischeKontemplationsicheinstellt, bedarfes der Schönheit des Objekts, das übersichselbsthinausweist, aberauch der Besonnenheit des Subjekts. DavonsollimFolgenden die Redesein.

\footnotetext{
${ }^{7}$ Vgl. W I, 218ff.,auch § 39, „Über das Erhabene“, hier S. 238.
} 


\section{Besonnenheit}

Zunächstmüssenwir auf den so schwerzuübersetzendenBegriff „Objektität““ zurückgreifen, die Schopenhauer mit der Idee und der Sichtbarkeit des in ErscheinungtretendenWesensselbstgleichgesetzt, indemer die IdeekennzeichnetalsadäquateWillens- und damitWesensobjektität, Objektivation „auf höchsterStufe“. Einzig die Idee hat die Eigenschaft, einederartangemessene, nämlich die ,ideale“ Objektivation des Willenszusein, dass das ErscheinungshaftenichtvomWesentlichenablenkt, sondernvielmehrzuihmführt, dass das Erscheinungshaftedurchschaubar und das Wesentlichesichtbarwird - und erkanntwerdenkann:

„Daß die richtigeanschaulicheAuffassung der Welt die Quelle der Weisheitist, nicht das abstrakteWissen, dies ista priorieinzusehn, daraus, daß die Erkenntniß der Ideen (siehe $3^{\text {tes }}$ Buch) eineanschaulicheist, ihreErkenntnißaber die höchsteWeisheitgiebt, nicht die Beobachtung des endlosenWechsels in der Zeit, noch der unzählbarenVielheitimRaum, da dieses allesnurErscheinung der Ideenist und nurbeachtenswerth, so lange man die Ideennichterkennt.“ (HN III, 100/ Foliant I, Nr. 57: Berliner MS, 1821)

Außer der IdeeimAllgemeinenbezeichnet der Begriff der Objektitätaberauch das Sichtbarsein und damit das Erkanntwerden des WillensimleiblichenIndividuum, z.B. mitdemSatz „Mein Leibist die ObjektitätmeinesWillens“; dortheißtesauch, „die Erkenntniß, welcheich von meinemWillenhabe [..., [ist] von der meinesLeibesnichtzutrennen“ (W I, 121). Damit ist eine Aussage getroffen, die die Identität des Meinigen, des je eigenen Leibes und Willens, postuliert. Die ursprünglichste Wesensbewusstheit, die gefühlte „Elementareinsicht“, dass ich Wille bin, ist also zugleich eine auf das individuelle Subjekt beschränkte, und eine allgemeine, da für jedes individuelle Subjekt geltende. Indem ich mich leibhaftig bewusst als mein Wille erfahre, weiß ich um die Idee meines individuellen Wesens als Einheit von Leib und Ich-Bewusstsein und erlebe, modern gesprochen, „ganzheitliches“ Selbstbewusstsein. Dieses Wissen um die Idee des Individuums ist letztlich gleichen Inhalts wie das durch ästhetische Kontemplation gewonnene: es ist die Gewissheit des in Vereinzelung erscheinenden, aber dennoch allumfassenden und allgegenwärtigen Wesens; allerdings eine gefühlte Gewissheit. 
Aus dem Zusammenwirken aller Erkenntnisvermögen und des idealen Objekts ergibt sich nun die besondere Bewusstseinsverfassung der Besonnenheit. Ich bin der Auffassung, dass sie weniger als Eigenschaft gedacht werden muss denn als Haltung, im Sinne einer aufmerksamen, reflektierten, integrativen Bewusstheit, aus der philosophische Erkenntnis wie auch (moralisches) Verhalten hervorgehen. Gerade in der besonnenen Haltung drückt sich die Idee aus, die nach Schopenhauers Ideenlehre die höchste Stufe der Willensobjektivationen ausmacht: die Idee des erkenntnisfähigen und vernunftbegabten Menschen, für den gilt:

„Wie nun, in unendlichen Abstufungen, die Deutlichkeit des Bewußtseyns sich steigert, tritt mehr und mehr die Besonnenheit ein [...]“(W II, 437)

Schon in seiner Dissertation spricht Schopenhauer von Besonnenheit in dieser allgemeinen Bedeutung:

„[...] Besonnenheit [...], also die Fähigkeit sich zu besinnen, zu sich zu kommen, ist eigentlich die Wurzel aller seiner theoretischen und praktischen Leistungen, durch welche der Mensch das Thier so sehr übertrifft [...]“(G, 117 / Diss)

Besonnenheit führt also zur „Besinnung“, auch zu verstehen als Be-Sinnung; der besonnene Mensch ist fähig, den Sinn der Welt wie den seiner selbst aus dem deutlichen Bewusstsein der reinen Anschauung heraus in sich zu finden, ,inne [zu] werden“. Es handelt sich damit bei der Besonnenheit um die adäquate Anwendung der menschlichen Vernunft selbst.

Um die erforderliche außergewöhnliche, die Vorstellung zur Einsicht in das metaphysische Prinzip transzendierende Abstraktionsleistung $\mathrm{zu}$ vollbringen, bedarf es Schopenhauer zufolge bekanntermaßen nicht allein der gefühlten „Elementareinsicht ${ }^{\star 8}$, dass ich meinem Wesen nach Wille bin, und deren Übertragung auf die Welt qua Analogieschluss, sondern einer besonderen inneren Haltung, die bildlich gesprochen eher einem inneren Salto zu gleichen scheint: Es bedarf der Besonnenheit, zu der nur die Distanzierung vom Fühlen und Wollen durch die Reflexion der „Elementareinsicht“ im Erkennen verhilft. Die Idee des Individuums enthält in sich die Überzeugung, dass der Mensch die Fähigkeit des Bewusstseins besitzt, sich mit seiner Erkenntnisfunktion zu identifizieren, d.h. rein

\footnotetext{
${ }^{8}$ Der Begriff „Elementareinsicht“ geht auf Rudolf Maltersfundamentaler Schopenhauer-Exegesezurück, auf die hierausdrücklichverwiesensei: Arthur Schopenhauer - Transzendentalphilosophie und Metaphysik des Willens. Stuttgart-Bad Cannstatt: frommann-holzboog, 1991, hierinsbesondere der Abschnitt,,Die Schwierigkeit der Verallgemeinerbarkeit der metaphysischenElementareinsicht“, 223-247.
} 
erkennendes Subjekt zu sein, für das sich der bloße Willen zum Leben als ein sich selbst erkennender, als Wille zur Selbst-Erkenntnis darstellt. Konkret stellt Schopenhauer sich diesen Zustand der Besonnenheit als Ergebnis asketischer Lebensführung vor, deren Ziel es ist, nur überlebensnotwendigen körperlichen Bedürfnissen Genüge $\mathrm{zu}$ tun, um Lust-Unlust-Empfindungen des Leibes weitgehend zu unterdrücken, die ja der Wille selbst sind, wie er im Bewusstsein vorkommt - kurz: als Ergebnis der Willensverneinung. ${ }^{9}$

Im 4. Buch beschreibt Schopenhauer daher folgerichtig, dass und warum die Besonnenheit dauerhaft und nachhaltig durch Verneinung des Willens in der Askese erreicht werden kann. Die maximale Reduktion der das Bewusstsein besetzenden Willensakte, die es - komplementär verstanden - zum wollenden auf Kosten des erkennenden machen, soll also die menschliche Erkenntnisfähigkeit optimieren. Die Verneinung des Willens entspricht einer Weigerung, die Macht der Äußerungen des erkenntnislosen Lebensdranges als vorrangig vor allen anderen Erfahrungen $\mathrm{Zu}$ akzeptieren.

ErstunterdieserVoraussetzungistsystematischephilosophische

(Vernunft-

)Erkenntnismöglich:

„Denneben Das, was die ChristlichenMystiker die Gnadenwirkung und Wiedergeburtnennen, istuns die einzigeunmittelbareAeußerung der Freiheit des Willens. [...] Die Möglichkeit der also sichäußerndenFreiheitist der größteVorzug des Menschen, der demThiereewigabgeht, weil die Besonnenheit der Vernunft, welche, unabhängigvomEindruck der Gegenwart, das Ganze des Lebensübersehnläßt, Bedingungderselbenist.“ (W I, 478; Sperrungenim Original, kursiveHervorhebung von mir, M. R.)

Doch man solltedarübernichtaußerAchtlassen, dass Schopenhauer die BesonnenheitnichtnuralseineHaltungbeschreibt, die Asketen und Heiligenvorbehaltenist, sondern die immerdortvorkommt, woIdeenerkenntnisstattfindet, die unsauch der Wahrheit und der Weisheitnäherbringt; sieentsprichteiner „Erkenntnißweise“, die

9 S. hierzu die Definition von Askese, W I, 449: „Jeneraber, der, das principium individuationisdurchschauend, das Wesen der Dinge an sich und dadurch das Ganzeerkennt, [...] siehtsichanallenStellenzugleich [...]. - SeinWillewendetsich, bejahtnichtmehrseineigenes, sich in der ErscheinungspiegelndesWesen, sondernverneintes. Das Phaenomen, wodurch dieses sichkundgiebt, ist der Uebergang von der TugendzurAskesis.“ Vgl. auch Ruffing, ,WillezurErkenntnis“, Kap. 4, besonders 128-140. 
übergefühlteGewissheithinausgeht, nämlich der Kunst, und die „Genialität“, oderBesonnenheit, erfordert:

Der Genius hat [... den vielhöhern Grad und die anhaltendereDauerjenerErkenntnißweisevoraus, welcheihnbeiderselben die Besonnenheitbehaltenlassen, die erfordertist, um das so Erkannte in einemwillkürlichenWerkzuwiederholen, welcheWiederholung das Kunstwerkist. (W I, 229; Hervorhebung von mir, M.R.)

Indem Schopenhauer erläutert, wie objektive Wahrheit und subjektive Wahrhaftigkeit in der ästhetischen Kontemplation zusammenkommen, entwickelt er ein philosophisches Selbst- und Weltverständnis, das seine „Beglaubigung in sich“ hat (W II, 533). Seine Theorie der ästhetischen Kontemplation macht die Welt als sich materialisierende und realisierende Selbsterkenntnis des Lebendigen, bzw. Erkenntnis des lebendigen Selbst, verstehbar. Unter dem Aspekt der Erkenntnistheorie ist sie somit Voraussetzung für „Weisheit“, womit Schopenhauer die philosophische Erläuterung des „wahren“ Selbst-Verständnisses des Menschen meint, das auf Besonnenheit beruht. In praktischer Hinsicht, als Ausdruck der Verneinung des eigenen Willens - und letztlich sogar des Willens überhaupt - ist sie Voraussetzung für eine Einstellung, aus der heraus allein moralisches Handeln möglich ist, eine Einstellung, die Schopenhauer auch „Güte der Gesinnung“ nennt: Sich selbst an die Stelle des anderen zu setzen und gewissermaßen auch an dessen Stelle, zu dessen Wohl, für ihn zu handeln. So ist das dritte Buch nicht nur die ,Summe‘, die Synthese, des ersten und des zweiten, sondern das Verständnis der ästhetischen Kontemplation ist - erkenntnistheoretisch - auch notwendige Voraussetzung des vierten Buches, in dem Schopenhauer seine Philosophie, verstanden als Weisheitslehre, erst zum Abschluss bringt. 